$\left(\mathrm{CO}_{2}\right)_{2}$ and $\left(\mathrm{N}_{2} \mathrm{O}\right)_{2}$ as small as $3 \mathrm{MHz}$, yielding an upper limit of $50 \mathrm{~ns}$ for the lifetime of the upper state. Simultaneously, DeLeon and Muenter have measured vibrationally excited $(\mathrm{HF})_{2}$ dimers and found comparable linewidths. The door is thus now wide open for the determination of dimer structures and intra-dimer dynamics.

\section{Applications}

Dimer predissociation can be achieved with the absorption of a single photon. If there are big differences between the spectra of molecules of different isotopic composition, the method would hold promise for applications in the future. The group of Manot and van den Bergh (Lausanne) who have been pursuing this line have recently come up with an elegant two-stage scheme. We discuss it by considering $\mathrm{SF}_{6}$ which can be considered to have two isotopomers $\left({ }^{32} \mathrm{~S}^{19} \mathrm{~F}_{6}\right.$ and $\left.{ }^{34} \mathrm{~S}{ }^{19} \mathrm{~F}_{6}\right)$. First, the $0.5 \%$ $\mathrm{SF}_{6}$ in $\mathrm{Ar}$ mixture is irradiated close to the nozzle (see Fig. 1) so that one monomeric isotopomer becomes excited and is inhibited from forming clusters (i.e. $\mathrm{SF}_{6}-\mathrm{Ar}_{n}$ ). Then, a second laser downstream predissociates those clusters that have been formed, and the fragments collected contain only the other isotopomer. Note that the first laser is tuned to induce absorption of a photon by one monomer, which possesses narrow and well defined absorption bands. On the other hand, the second laser does not need to distinguish separate absorption bands for different isotopomer dimers; only one type is present due to the inhibition of the formation of the other by the first laser. The great advantage of the Lausanne method is that only one (single expensive) IR photon is needed per molecule to achieve enrichment (in contrast to the MPD schemes). Another application stems from the unique possibility of being able to identify and select certain dimers by their spectra (e.g. $\left(\mathrm{SF}_{6}\right)_{2}$ and $\mathrm{C}_{2} \mathrm{H}_{4}-\mathrm{Ne}$, the exceptional cases). By tuning the mass spectrometer detector to different masses, the fragmentation has been determined quantitatively. For $\left(\mathrm{SF}_{6}\right)_{2}$ it has been found by the Nijmegen group that the dimers show up on the mass of $\mathrm{SF}_{5}^{+}$at least a hundred times more frequently than on the dimer ion mass $\mathrm{SF}_{6}-\mathrm{SF}_{5}^{+}$.

\section{Further Reading}

Attention is drawn to two comprehensive articles:

Gentry W.R., "Vibrationally Excited States of Polyatomic van der Waals Molecules: Lifetimes and Decay Mechanisms", ACS Symposium Series 263 (1984) 289-303. Janda K.C., "Predissociation of Polyatomic van der Waals Molecules", Adv. Chem. Phys. 60 (1985) in print.

\title{
Atomic Processes in Astronomy
}

\section{M.J. Seaton, London}

(University College)

The astronomer is often concerned with the behaviour of matter under conditions which are unusual from the standpoint of the laboratory physicist, either because they are very simple or because they are very extreme. In either case, studies of such conditions have led to important advances in basic physics. Many examples could be given: studies of the laws of dynamics and gravitation, of ultra-high densities, and of thermonuclear energy generation. I consider some examples from atomic physics.

Many astronomical spectra differ from laboratory spectra as a consequence of being produced at very low densities (the pressure in a typical gaseous nebula is of order $10^{-14} \mathrm{~atm}$, that in the interstellar medium about $10^{-20} \mathrm{~atm}$ ) and this simplifies their interpretation. The way in which strengths of lines depend on density can be understood by considering carbon-like ions, which have ground configurations $1 \mathrm{~s}^{2}$ $2 s^{2} 2 p^{2}$ with three terms, ${ }^{3} \mathrm{P},{ }^{1} \mathrm{D}$ and ${ }^{1} \mathrm{~S}$. The energy-level diagram for the carbonlike ion $\mathrm{O}^{2+}$, which gives strong lines in many astronomical spectra, is shown in Fig. 1. Since the ground configuration terms all have the same parity, radiative transitions between them cannot occur by electric-dipole radiation. Such levels are said to be "metastable" and the transitions to be "forbidden", but they can take place by electric-quadrupole or magnetic-dipole radiation with probabilities of the order of $1 \mathrm{~s}^{-1}$ compared with $10^{9}$ $\mathrm{s}^{-1}$ for "allowed" lines. In radiation from laboratory sources the forbidden lines are very weak, and are rarely seen, but in the spectra of gaseous nebulae they are often the strongest features observed. The number of photons emitted per unit time in a transition $2 \rightarrow 1$ is given, in all cases, by

$$
P_{21}=N_{2} A_{21}
$$

where $N_{2}$ is the number of atoms in the upper level and $A_{2}$, the radiative transition probability. At densities of laboratory sources, the probabilities of transitions due to collisions are much larger than $A_{21} \cong 1 \mathrm{~s}^{-1}$. To a good approximation, the relative populations of the ground and metastable levels are given by the Boltzmann equation, and the forbidden lines are very weak, compared with other lines, because $A_{2}$ in (1) is very small. At the low densities of nebulae, collisions are much less frequent and are mainly due to electron impacts, which produce both excitation and de-excitation. Let $N_{e}$ be the electron density and $q_{i j} N_{\mathrm{e}}$ the probability of a collisional $\mathrm{i} \rightarrow \mathrm{j}$ transition $\left\langle q_{\mathrm{ij}}=\left\langle v_{\mathrm{i}} Q_{\mathrm{ij}}\right\rangle\right.$ where $v_{i}$ is the velocity of the incident electron, $Q_{i j}$ the collision cross-section, and the average is over the velocity distribution). At a critical density $N_{e}$ (c), such that $q_{21} N_{e}$ (c) $=A_{21}$, the probability for collisional de-excitation equals that for de-excitation by emission of radiation. For the forbidden lines, typical values of $N_{e}$ (c) are of order $10^{4} \mathrm{~cm}^{-3}$. For $N_{\mathrm{e}}<<N_{\mathrm{e}}$ (c) effectively every excitation by electron impact is followed by emission of radiation, giving

$$
P_{21}=N_{1} q_{12} N_{\mathrm{e}}
$$

which is independent of $A_{21}$. Densities $N_{\mathrm{e}} \leq N_{\mathrm{e}}$ (c) frequently occur in gaseous nebulae and this explains why the forbidden lines can be the strongest features in their spectra.

The observed spectra provide the astronomer's only means of estimating temperatures and densities in nebulae. If the condition $N_{\mathrm{e}}<<N_{\mathrm{e}}$ (c) can be assumed for two lines (such as the $\lambda=$ $5007 \AA$ and $4363 \AA$ lines of Fig. 1), the ratio of their intensities will depend only on the ratio of their excitation rates, and
Fig. 1 - Partial energy-level diagram for $\mathrm{O}^{2}+$ (not to scale) showing lines observed in the spectra of nebulae at $I R$, optical and UV wavelengths.

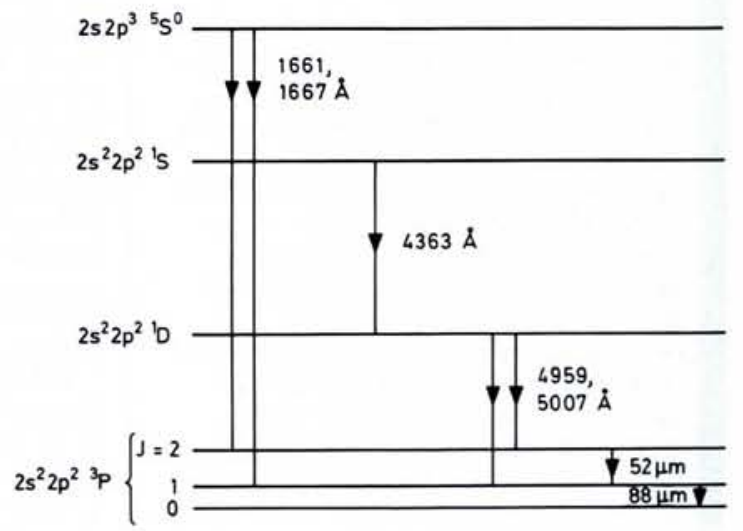


hence only on electron temperature $T_{e}$. It is fortunate that many nebulae have densities comparable with $N_{e}$ (c) for some of the forbidden lines, and hence intensity ratios which depend on both $T_{\mathrm{e}}$ and $N_{\mathrm{e}}$. From a number of observed ratios both $T_{\mathrm{e}}$ and $N_{\mathrm{e}}$ can be deduced, and once those parameters are known, one can deduce the chemical composition of the emitting gas.

\section{Extending the Wavelength Range}

Observations made above the absorbing layers in the Earth's atmosphere have greatly enriched our knowledge of astronomy. For objects outside the solar system there is a strong absorption of radiation, at wavelengths $\lambda \leq 912 \AA$, due to photo-ionisation of interstellar atomic hydrogen, but as the cross-section falls off as $\lambda^{3}$, observations again become possible at $X$-ray (XR) wavelengths. In the more readily accessible ultra-violet (UV) region, $\lambda>912 \AA$, observed spectra contain many intercombination lines (allowed for electric-dipole radiation when account is taken of departures from LS coupling) and resonance lines such as C IV $2 p \rightarrow 2$ s $\lambda=1548,1551$ $\AA$. In the infra-red one observes transitions between atomic fine-structure levels. Fig. 1 shows the lines of $\mathrm{O}^{2+}$ observed from nebulae at IR, optical and UV wavelengths.

In his 1913 paper on the $\mathrm{H}$ atom, N. Bohr discussed transitions between highly-excited states, $n \rightarrow(n-\Delta n)$ with $n$ large and $\Delta n$ small, which should occur in the region which he referred to as "the extreme ultra-red". Such transitions would be difficult to detect in the laboratory, since the radii of the orbits are very large, but they have been detected by radio astronomers. The highly-excited states are populated by recombination and significant population inversions can occur. These lead to line intensification by maser action and, even at the very low densities in nebulae, collisional effects can cause line-broadening. Active work on observing the "radio recombination lines" continues at the Max-Planck-Institut für Radioastronomie in Bonn and at Jodrell Bank in Cheshire.

Once one can get above the Earth's atmosphere, radiation from the Sun can be observed over a very extended range of wavelengths, out into the far UV and XR regions. The greater part of the solar energy output is, however, in the optical and near IR, and comes from the photosphere or solar "surface" which is at about $6000 \mathrm{~K}$. The corona, first observed during eclipses, extends out to several times the photospheric radius and the chromosphere is a transition region bet-
AP ACADEMIC PRESS AP

\section{SUPERLATTICES AND MICROSTRUGTURES}

\author{
Editor in Chief: John D. Dow \\ Department of Physics, University of \\ Notre Dame, Indiana, USA \\ Volume 1, 1985, Bi-monthly \\ £75.00 (UK only)/\$132.00 (Overseas)
}

Superlattices and Microstructures is an interdisciplinary journal devoted to the physics, chemistry, materials science, and electrical engineering of submicron structures not only in semiconductors but also in materials with metallic, insulating and superconducting properties. It publishes original articles on the electronic and mechanical properties of synthetic structures as well as the transport and dynamical properties of heterogeneous solids. Papers are encouraged on related topics in surface science, devices physics, crystal growth, interface phenomena, and the many applications of submicron structures.

\section{Sample copies available from Journals Marketing Dept.
(Specify journal title).}

\section{4-28 OVAL ROAD, LONDON, NWI 7DX ORLANDO, FLORIDA 32887}

ween the photosphere and the corona. The first definitive evidence that the corona has a high temperature, $T \geq 10^{6} \mathrm{~K}$, came from the identification by the Swedish spectroscopist B. Edlén that a number of lines in coronal optical spectra are due to transitions in highlyionised atoms (such as $\mathrm{Fe}^{9+}$ and $\mathrm{Fe}^{13+}$ ). Subsequent studies of the corona laid the foundations for much of our present knowledge of the physics of hot plasmas. By far the richest parts of coronal and chromospheric spectra occur, of course, at UV and XR wavelengths. In recent years, coronae and chromospheres have also been observed in stars other than the Sun.

\section{Suns and Stars}

Spectra of nebulae and coronae are dominated by bright emission lines, essentially because the sources are optically thin, i.e. emitted quanta can escape without re-absorption. Photospheres, on the other hand, are optically thick and have continuous spectra with lines in absorption. Photospheres are not in thermodynamic equilibrium because radiation can eventually escape, but at each point within them it is a very good approximation to assume a local kinetic temperature. In earlier work by, amongst others, M.G.J. Minnaert at Utrecht and A. Unsöld at Kiel the assumption was made (known as local thermodynamic equilibrium or LTE) that thermodynamic laws could be used to calculate ionisation equilibria and level populations. With that assumption, quantitative spectrum analysis requires atomic data only for radiative processes. For non-LTE analyses, which were pioneered by $D$. Mihalas and others in the USA, all collisional processes must also be considered. Non-LTE effects are important in hot stars but for cooler stars LTE is generally a good approximation. Particularly active research in analysis of stellar spectra is pursued at the German Universities of Kiel, Heidelberg and Munich.

The analysis of astronomical spectra requires large amounts of atomic data

\section{THE UNIVERSITY OF MANCHESTER DEPARTMENT OF PHYSICS}

\section{Postdoctoral Research Associate in Experimental Nuclear Structure Physics}

Applications are invited for the above post funded by the SERC and tenable immediately until 31 January, 1987. The successful candidate will be expected to initiate and assist with research at the Nuclear Structure Facility, a 20 MV tandem Van de Graaff, which is now operational at the Daresbury Laboratory. Applicants are expected to hold a Ph.D. degree in Nuclear Structure Physics and should have an aptitude for pursuing research in experimental physics. Salary range $£ 7,190-£ 8,530$ p.a. (under review). Superannuation.

Applications with full c.v. and names of two referees should be sent as soon as possible to Dr. R. Chapman, Department of Physics, The University, Manchester M13 9PL from whom further details may be obtained. 


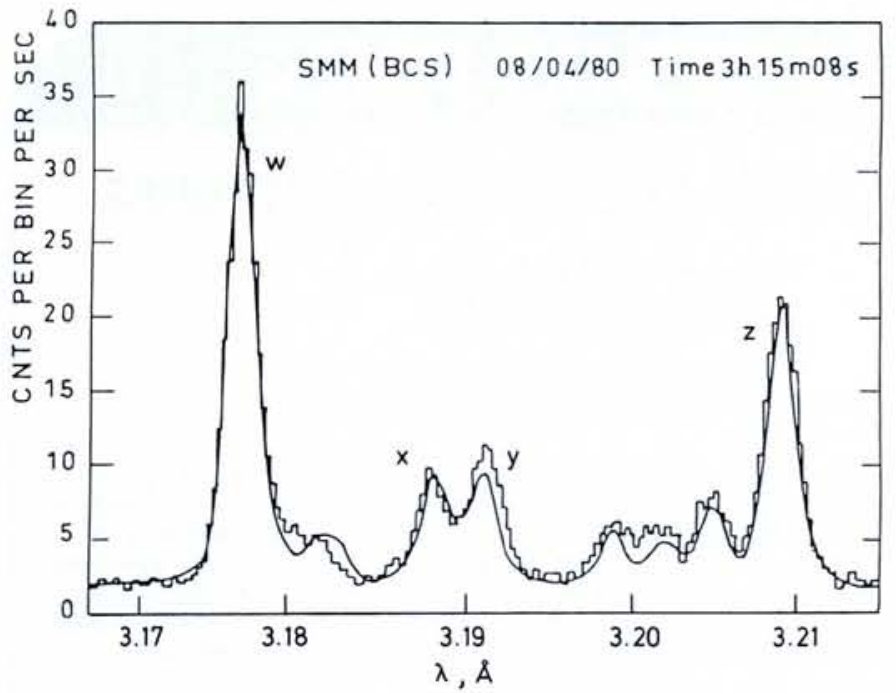

Fig. $2-A$ solar $X R$ spectrum in the vicinity of the resonance line of He-like Ca, $\mathrm{Ca}^{18+}$. The line identifications are discussed in the text.

and, since the physical conditions are very different from those obtainable in the laboratory, much of the data must come from quantum mechanical calculations (experimental checks are, of course, always highly desirable). The required data are: radiative transition probabilities (usually expressed as oscillator strengths, or $f$-values); pressurebroadening parameters; cross-sections for photo-ionisation and the inverse process of radiative recombination, freefree transitions (thermal bremsstrahlung) and collisional excitation and ionisation; and rates for charge-transfer reactions.

The spectral distribution of solar photospheric radiation is largely determined by photo-detachment from the $\mathrm{H}^{-}$ion, $\mathrm{H}^{-}+h v=\mathrm{H}+\mathrm{e}$, and by the free-free process $\mathrm{H}+\mathrm{e}+h v=\mathrm{H}+$ e. Elaborate calculations for these processes, involving only two electrons, give results which should be accurate to $1 \%$ and which are in agreement with experimental results. Accurate calculations of $f$ values are possible for lighter atoms but not yet possible for heavier atoms such as Fe which is of interest because of its relatively high cosmic abundance. Accurate measurements of $\mathrm{Fe} \mid \mathrm{f}$-values have been made by D.E. Blackwell and collaborators at Oxford and by M.C.E. Huber and E.F. Tubbs at Harvard. Relative values correct to about $1 \%$ are obtained using absorption techniques and put on an absolute scale, are correct to $10 \%$, using absolute measurements for one or two strong lines. The pressurebroadening of solar lines is mainly due to $\mathrm{H}$-atom collisions. Laboratory studies can be made of broadening by collisions with rare-gas atoms but similar work with atomic hydrogen would be very difficult. Astronomers use "damping constants" for $\mathrm{H}$-atom collisions deduced from analyses of the solar spectrum. Atmospheres of hot stars have significant numbers of $\mathrm{H}$ atoms in excited states and hence prominent $\mathrm{HI}$ absorption lines in their spectra. In these atmospheres most of the atoms are ionised and electric fields due to the charged particles give broadening of $\mathrm{HI}$ lines by the Stark effect (which is linear in hydrogenic systems because of $\ell$-degeneracy). Developments in the theory of this process have been of importance to astronomers, since the $\mathrm{HI}$ line profiles provide information on pressure and hence on gravitational forces.

Profiles of spectral lines from the corona are determined by thermal Doppler broadening and provide a measure of temperature. Until about 20 years ago, the temperatures from line-profiles were found to be higher than those deduced from studies of the ionisation-equilibrium balance between collisional ionisation and radiative recombination. $A$. Burgess resolved the problem by showing that large enhancements in recombination rates result from taking account of the di-electronic process

$$
\mathrm{X}^{+}+\mathrm{e}=\mathrm{X}^{* *} \rightarrow \mathrm{X}^{*}+h v
$$

where $X^{* *}$ is an unstable state with two electrons excited. There is much current interest in experimental and theoretical studies of such processes. Some remarkable solar XR spectra have been obtained in the vicinities of resonance lines of $\mathrm{He}$-like ions. Fig. 2 gives an example for a region near $3 \AA$. The features marked $w$, $\mathrm{x}, \mathrm{y}$ and $\mathrm{z}$ are due to transitions in $\mathrm{He}$-like $\mathrm{Ca}, \mathrm{Ca}^{1} 8+: \mathrm{w}$ is the resonance line, $1 s 2 p^{1} \mathrm{P}_{1}^{O} \rightarrow 1 \mathrm{~s}^{2}{ }^{1} \mathrm{~S}_{0} ; \mathrm{y}$ the intercombination line, $1 s 2 p{ }^{3} p_{1}^{0} \rightarrow 1 s^{2}{ }^{1} S_{0} ; x$ the magnetic quadrupole line $1 \mathrm{~s} 2 \mathrm{p}{ }^{3} \mathrm{p}_{2}^{\mathrm{O}} \rightarrow$ $1 \mathrm{~s}^{2}{ }^{1} \mathrm{~S}_{0}$; and $\mathrm{z}$ the relativistic magnetic dipole line $1 s 2 s{ }^{3} S_{1}^{0} \rightarrow 1 s^{2}{ }^{1} S_{0}$. Many other features are also observed and are due to "di-electronic satellites", transitions of the type $1 \mathrm{~s} 2 \mathrm{pn} \ell \rightarrow 1 \mathrm{~s}^{2} \mathrm{n} \ell$. Elaborate modelling is used to fit the observed spectra and is particularly valuable for the study of solar-flare events.

\section{Close-coupling Calculations}

The astronomer is usually concerned with plasmas for which the mean kinetic energy of the electrons, $k T=<1 / 2$ $m v^{2}>$, is small compared with the ionisation potentials, $l$, of the abundant ions, and is therefore interested in nearthreshold electron-impact excitations of the lower energy levels of atomic ions. This is fortunate, since it means that the close-coupling approximation can be used. For a one-electron target the approximation employs expansions of the type

$\psi=\sum_{n=1}^{N}\left\{\psi_{n}\left(x_{1}\right) \theta_{n}\left(x_{2}\right)-\psi_{n}\left(x_{2}\right) \theta_{n}\left(x_{1}\right)\right\}$

where $x_{1}, x_{2}$ are co-ordinates of the electrons, the $\psi_{i}$ are target states and the states $\theta_{i}$ are functions for the colliding electron and are calculated on solving a system of coupled integrodifferential equations. So long as the conditions $1 / 2 m v^{2}<</$ is satisfied, the number of terms $N$, included in (4) can be kept reasonably small and the approximation is both practicable and accurate. For higher energies, electronimpact calculations are much more difficult. In particular, for near-threshold electron-impact ionisation, accurate methods of calculation have not yet been developed and astronomers use results of approximate calculations, experimental results when available, and scaling laws suggested by semi-classical theories.

Many accurate calculations of electron-impact excitation cross-sections have been made in recent years. Large computer-program packages have been developed at University College London (UCL) and by P.G. Burke and collaborators at The Queen's University of Belfast (QUB). Research groups at Paris, Nice,

Fig. 3 - Partial energy-level diagram for $\mathrm{C}^{2+}$, showing lines observed in astronomical spectra. $2 p^{2} / s$

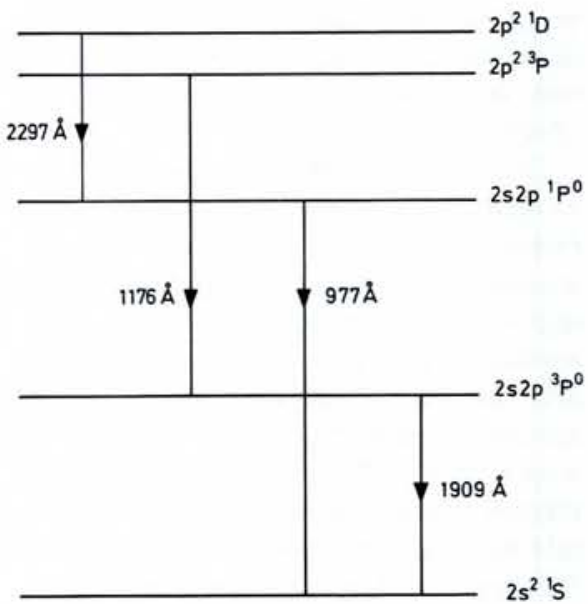


Zurich and Munich have been actively engaged in the work, as well as groups in the USA. Many of the cross-sections are found to have complex resonance structures due to the formation of compound states,

$$
\mathrm{X}_{\mathrm{i}}+\mathrm{e} \rightarrow \mathrm{X}^{* *} \rightarrow \mathrm{X}_{\mathrm{i}}+\mathrm{e} \text {. }
$$

A case study is provided by work on $\mathrm{C}^{2+}$, for which an energy-level diagram is given in Fig. 3. The first detection by astronomers of the inter-combination line ${ }^{3} \mathrm{P}^{\circ} \rightarrow{ }^{1} \mathrm{~S}, \lambda=1909 \AA$, was in the spectra of quasars, which have redshifts so large that UV features are shifted into the optical region. In the early 1960s, the excitation cross-section for $\lambda=1909 \AA$ was uncertain, even as to order of magnitude, and realising the importance of the problem for quasar studies, D.E. Osterbrock, of the Lick Observatory, made the first $\mathrm{C}^{2+}$ closecoupling calculations with inclusion of the three lowest $\mathrm{C}^{2+}$ levels. In 1973 the UCL close-coupling code was used by D.R. Flower and J.M. Launay, at the Paris Observatory, to make calculations that included the six levels shown in Fig. 3 and they obtained results correct to within $25 \%$. The $\mathrm{CIII}$ allowed lines at $\lambda$ $=1176 \AA$ and $977 \AA$ are observed in the solar spectrum, as well as the intercombination line at $1909 \AA$. Since the transition probability for this is fairly small (about $100 \mathrm{~s}^{-1}$ ), measured relative line
Fig. 4 - Cross-sections for excitation of the $C^{2}+$ lines at $1909 \AA$ and $977 \AA$ (see Fig. 3), from calculations by K.A. Berrington, P.G. Burke, P.L. Dufton and A.E. Kingston (J. Phys. B, 10, (1977) 1465. The complicated resonance structures are due to processes of the type (5).
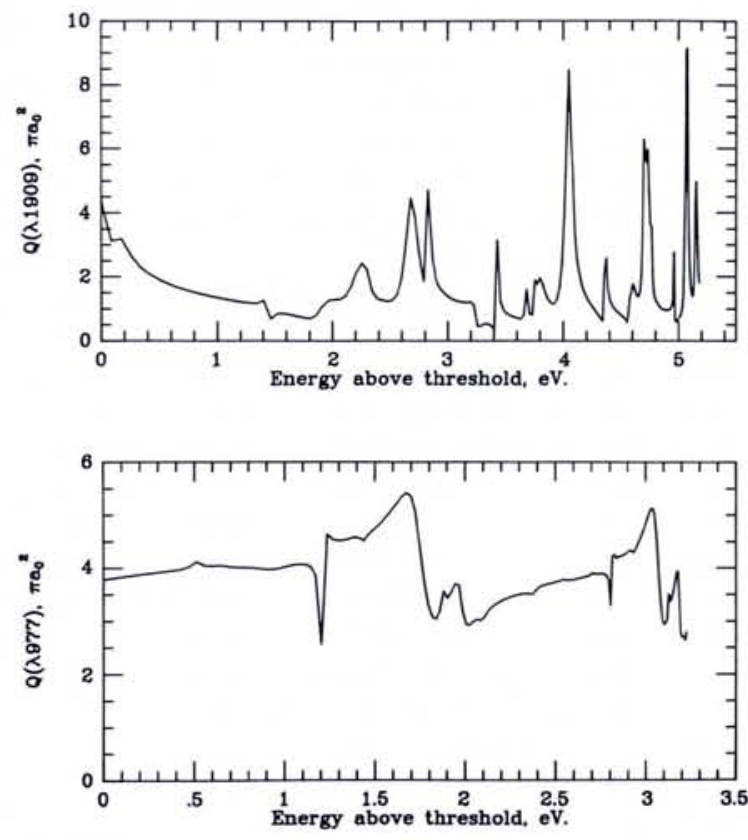

intensities depend on density. The densities deduced for the emitting region differed significantly from values for standard models of the solar atmosphere. C. Jordan suggested that more accurate atomic data were required, and very elaborate calculations were made in 1977 by the QUB group and gave results which eliminated the most worrying discrepancies in density deter- minations. Fig. 4 shows the complexity of the resonances in the $\mathrm{C}^{2+}$ crosssections. Yet another CIII line, $\lambda=2297$ $\AA$ (see Fig. 3), is observed in the spectra of nebulae and of novae. Its upper state, $2 p^{2} D$, is too high for collisional excitation and P.J. Storey has shown it to be populated by di-electronic recombination involving low-lying doubly-excited states.
For your easy and quick access to Chinese physics: Chinese

\section{Physics Letters}

Editor: HUANG Zu-qia, Beijing

Associate Editors: LI Yin-yuan, Beijing; HAO Bai-lin, Beijing; GAN Zi-zhao, Beijing; WANG Ding-sheng, Beijing

The new monthly journal Chinese Physics Letters (CPL), edited by the Chinese Physical Society as a letters section of Acta Physica Sinica, is devoted to the rapid publication of short reports of important research in all fields of physics. It is published in English to further international exchange. For quickness the articles appear only in English - they are not translations of previously published chinese papers.

CPL not only accepts papers on important fundamental research but also accepts a substantial portion from applied physics, cross-disciplinary studies, and even papers of general interest to a wider readership. Papers published in CPL differ from those published in the Brief Reports section of other physics journals in China (for example, Acta Physica Sinica): the former are usually of greater importance and will be followed by a complete report elsewhere, while the latter need not be described further in a long paper. The editors lay stress on the scientific soundness of a paper - the validity of the scientific content of the text and the importance of the results. Besides this, the general interest of a paper will also be considered.

This journal is published by Science Press, Academia Sinica. Each issue contains not fewer than 12 papers (48 printed pages). Two issues have been published in 1984. In 1985, CPL appears as a monthly journal.

Subscription Information:

ISSN: pending Title No. 375

1984, vol. 1 ( 2 issues): DM 57,- plus carriage charges 1985, vol. 2 (12 issues): DM 338,- plus carriage charges Detailed information and/or sample copy available from your bookseller or from the publisher.

Pcience Press, Beijing

Distributed by:

Springer-Verlag Berlin Heidelberg New York Tokyo

Tiergartenstr. 17, D-6900 Heidelberg 1 or 175 Fifth Ave., New York, NY 10010, USA or 37-3, Hongo 3-chome, Bunkyo-ku, Tokyo 113, Japan 


\section{A Curious Paradox}

Allowance for di-electronic recombination removes some discrepancies between observations and earlier calculations of the ionisation equilibria in nebulae. Others have been removed by taking account of charge-transfer with hydrogen, $\mathrm{X}^{+}+\mathrm{H}=\mathrm{X}+\mathrm{H}^{+}$, for which empirical studies have been made by $D$. Péquignot of the Paris Observatory and quantal calculations have been made by A. Dalgarno and collaborators at Harvard. In nebulae, most of the hydrogen is ionised but it is a curious paradox lexplained in terms of the $\lambda^{3}$ dependence of the hydrogen photo-ionisation cross-section) that the fraction of neutral $\mathrm{H}$ can be larger for a high-excitation nebula, photo-ionised by a very hot star. The charge-transfer process is found to be particularly important in nebulae of high excitation.

\section{Further Reading}

Blackwell D.E., "Stellar Atmospheres and Laboratory Astrophysics", Quart. J. R. Astr. Soc., 15 (1984) 224.

Atoms in Astrophysics, ed. P.G. Burke, W.B. Eissner, D.G. Hummer and I.C. Percival (Plenum Press) 1983.

Seaton M.J., "Atoms, Astronomy and Aeronomy", Quart. J. R. Astr. Soc., 23 (1982) 2.

\section{Hewlett-Packard Europhysics Prize}

The 1985 Hewlett-Packard Europhysics Prize has been awarded equally to Dr. Jens A. Als-Nielsen of the Risø National Laboratory, Roskilde, Denmark and Dr. Michael Pepper of the Cavendish Laboratory, Cambridge and the GEC Hirst Research Centre, Wembley, England, for "their contributions to the experimental study of low-dimensional physics". The presentation will be made at the 5th CMD Conference in Berlin (see above).

\section{5th EPS CMD General Conference in Berlin (West)}

The annual general conferences of the Condensed Matter Division are developing very successfully.

After the "new beginnings" with the first general conference in Antwerp (1980) (see Europhysics News, May, 1980) with over 600 participants, the question was whether such a Conference could be held on a regular basis as a kind of European counterpart to the March Meeting of the American Physical Society. Those that followed in Manchester (1982), Lausanne (1983), Den Haag (1984) have confirmed the need. The number of participants has remained at over 600 .

The 1984 Conference in Den Haag with Prof. F. Mueller as Chairman and Prof. P. Wyder as Chairman of the Programme Committee offered outstanding plenary talks and symposia, starting with R. Schrieffer's talk on solitons in condensed matter and closing with $\mathrm{G}$. 't Hooft's talk which reviewed techniques and concepts common to condensed matter physics and elementary particle theory. Other plenary speakers were Siegbahn, de Gennes, Benedek, Geballe, Hilsum, Binnig. Several of the symposia created a great deal of enthusiasm, and in particular the one on the quantum Hall effect (von Klitzing and fractional).

The 5th General Conference will take place in Berlin from March 18-22, 1985. Sessions will be held in the buildings of the Physics Department of the Technical University in Berlin - Charlottenberg. Just as The Institute of Physics did in 1982, the German Physical Society has agreed to cancel its 1985 "Frühjahrstagung" to act as host for the organization of the Conference.

Under the chairmanship of Prof. J. Treusch and with Prof. M. Cardona as Programme Committee Chairman, the 5 th General Conference holds great promise with close to 1000 contributed papers and an exciting plenary programme and symposia on recent developments. The number of suggestions which were made to the Programme Committee for invited papers was indeed impressive; research in condensed matter physics is doing well in Europe.

We look forward to a great meeting.

\section{J.T. Devreese}

Chairman of the Condensed Matter Division

\section{University of Antwerp Rijksuniversitair Centrum}

Chair for Theoretical and Mathematical Physics

\section{Post-doctoral Research Associate}

There is a vacancy for a theoretical physicist or chemist to join the theoretical physics research group which is involved in the detailed investigation of non-adiabatic effects in molecules. The main task of the applicant will be to extend existing computer codes for the treatment of near electronic degeneracies.

Applicants should have a good knowledge of the quantum theory of molecules and thorough experience with large scale electronic structure calculations.

The appointment will be made for the period of 1 year at the level of post-doctoral research associate with a salary of about $950.000 \mathrm{BF}$ per annum.

Applications with curriculum vitae (and major publications) should be sent as soon as possible to

Prof. Dr. P. VAN LEUVEN, Dienst Teoretische en Wiskundige Natuurkunde Rijksuniversitair Centrum Antwerpen, Groenenborgerlaan 171, B - 2020 Antwerpen

EPS Divisions, Sections and Group

Astronomy and Astrophysics Division Solar Physics Section

Atomic and Molecular Physics Division

Atomic Spectroscopy Section

Chemical Physics

Chemical Physics
Electronic and Atomic Collisions

Molecular Physics

Computational Physics Group

Condensed Matter Division

Liquids Section

Low Temperature Physics Section

Macromolecular Physics

Magnetism

Magnetism

Serniconductors and Insulators

Surfaces and Interfaces

High Energy \& Particle Physics Division

Nuclear Physics Division

Optics Division

Plasma Physics Division

Quantum Electronics Division
Europhysics News is the official journal of the European Physical Society which comprises 29 National Societies, Academies and Group, over 3500 Individual Mem. bers and 66 Associate Members. Governing bodies of EPS are the General Meeting. Council and an elected Executive Committee responsible for detailed policy. EPS promotes the collaboration of physicists throughout Europe, organising and harmonising conferences and publications, improving physics education, encouraging physics applications, awarding scholarships to sponsored schools in Erice. EPS publishes in addition to EN, Europhysics Conference Abstracts, E. Ed. News and, in collaboration with The Institute of Physics (UK), the European Journal of Physics. Individual Members receive EN free of charge (price to institutions: Sw.Fr. $90 .-/ a)$, rebates on the price of many publications and on conference fees. Annual EPS membership fee for Individual Members belonging to an EPS member society is: Sw.Fr. 40,-; independent members: Sw.Fr. 120.-; members of a Collaborating Society, e.g. the American Physical Society: Sw.Fr. 50- (\$25).

\author{
Editor: E.N. Shaw \\ Meetings Compilation: W.S. Newman \\ Editorial Board: \\ K. Appert, A. Baratoff, B. Jacrot. \\ G.R. Macleod, M. Mayor, J. Muller \\ Editorial and Advertising Office at the EPS \\ Secretariat \\ Address: EUROPEAN PHYSICAL SOCIETY \\ P.O. Box 69. \\ CH-1213 Petit-Lancy 2 \\ Switzerland \\ Telephone: Geneva (22) 931130 \\ Telex: 423455 dema ch \\ Cables: europhys genève \\ Printed by: Pfirter frères s: \\ CH-1213 Petit-Lancy/Switzerland
}

